\title{
Meteorological factors affect the hand, foot, and mouth disease epidemic in Qingdao, China, 2007-2014
}

\author{
F. C. JIANG ${ }^{1} \dagger$, F. YANG ${ }^{2} \dagger$, L. $\mathrm{CHEN}^{2} \dagger$, J. JIA ${ }^{1}$, Y. L. HAN ${ }^{1}$, B. HAO ${ }^{1}$ AND \\ G. W. $\mathrm{CAO}^{2 *}$ \\ ${ }^{1}$ Department of Acute Infectious Diseases, Municipal Centre of Disease Control and Prevention of Qingdao, \\ Qingdao, China \\ ${ }^{2}$ Department of Epidemiology, Second Military Medical University, Shanghai, China
}

Received 18 November 2015; Final revision 24 February 2016; Accepted 2 March 2016; first published online 28 March 2016

\section{SUMMARY}

Hand, foot, and mouth disease (HFMD) has caused public health concerns worldwide. We aimed to investigate the effect of meteorological factors on the HFMD epidemic in Qingdao, a port city in China. A total of 78641 cases were reported in Qingdao between January 2007 and December 2014. Of those, 71084 (90.39\%) occurred in children aged 0-5 years, with an incidence of 1691.2/100000. The incidence increased from early spring, peaked between spring and summer, and decreased in late summer. Aetiological agents in all severe cases and selected mild cases were characterized by examining throat swabs. Except for enterovirus 71 (EV71) and coxsackievirus A16 (CA16), other EVs caused $>50 \%$ of the HFMD cases between 2011 and 2014. EV71 was more frequent in the offpeak months than in the peak months and prone to causing more severe cases compared to CA16 $\left(\chi^{2}=46 \cdot 3, P<0 \cdot 001\right)$. CA10 caused more severe HFMD than did CA6 $\left(\chi^{2}=20 \cdot 49, P<0 \cdot 001\right)$ and all non-CA10 EVs $\left(\chi^{2}=41 \cdot 01, P<0 \cdot 001\right)$. Community-derived HFMD cases accounted for $65 \cdot 11 \%$. Spearman rank correlation analysis showed that HFMD incidence in children aged $0-5$ years was positively correlated with atmospheric temperature $\left(r_{s}=0 \cdot 77, P<0 \cdot 001\right)$, relative humidity $\left(r_{s}=\right.$ $0.507, P<0.001)$, and precipitation $\left(r_{s}=0.328, P<0.001\right)$. Climate changes and CA10 surveillance in communities should be integrated into the current prophylactic programme.

Key words: Coxsackievirus A6, coxsackievirus A10, hand, foot, and mouth disease (HFMD), human enterovirus 71, meteorological factors.

\section{INTRODUCTION}

Hand, foot, and mouth disease (HFMD), a communicable disease that often affects children aged $0-5$ years, poses a severe threat to children's health. Highly contagious enteroviruses (EVs) including human enterovirus 71 (EV71), coxsackievirus A16 (CA16), CA6, and

\footnotetext{
* Author for correspondence: Professor Guang-wen Cao, Department of Epidemiology, Second Military Medical University, Shanghai, 200433, China.

(Email: gcao@smmu.edu.cn)

$\dagger$ These authors contributed equally to this work.
}

CA10 have been proven to be the major aetiological factors [1-3]. EV71 is one of the aetiological viruses and often causes severe, sometimes fatal illness in East Asia [4-8]. HFMD is more frequent in summer than in the other seasons and is more frequent in hot areas than in cold areas [9-11]. Recent studies carried out in China indicated that the HFMD epidemic had spatiotemporal characteristics and that annual average temperature, relative humidity, and sunshine hours were positively related to the HFMD epidemic in different geographical areas, especially in warm areas [11-14]. However, the effects of weekly meteorological variables 
on the real-time HFMD epidemic in northern cold areas remain largely unknown. Understanding the effect of meteorological factors on the HFMD epidemic is particularly important for developing suitable prophylactic programmes in the context of climate change.

Qingdao is one of the largest tourist port cities in China with a population of $\sim 8715100$ (2010 census). It is located in the central section of the northern coastline of China $\left(35^{\circ} 35^{\prime}-37^{\circ} 09^{\prime} \mathrm{N}, 119^{\circ} 30^{\prime}-121^{\circ}\right.$ $\left.00^{\prime} \mathrm{E}\right)$ and belongs to the northern temperate maritime monsoon climate zone characterized by a hot, humid summer and a cold, windy winter. Qingdao has four distinctive seasons: spring (11 April-30 June), summer (1 July-10 September), autumn (11 September-10 November), and winter (11 November-10 April), which is very suitable to investigate the effects of meteorological factors on the HFMD epidemic.

\section{METHODS}

\section{Clinical and meteorological information}

From 1 January 2007, all HFMD cases were notified to the Municipal Centre for Disease Control and Prevention (CDC) of Qingdao via the National Notifiable Disease Reporting System (NNDRS) by medical practitioners. From 1 January 2009, the diagnostic criteria of mild and severe HFMD cases were implemented according to the 'Diagnosis and Treatment Guidelines of HFMD' formulated by the Ministry of Health in 2008 when HFMD was ranked as the 38th notifiable disease (http://www.moh.gov.cn/ publicfiles/business/htmlfiles/mohyzs/s3585/200812/ 38494.htm), as described previously [8]. Briefly, a mild case was characterized by mucocutaneous papulovesicular lesions on the hands, feet, mouth, and buttocks with or without fever, which resolved spontaneously within 7 days; a severe case was diagnosed when an exanthematous disease with cardiopulmonary complications and/or encephalitis was present. A laboratorydiagnosed case met one of the following criteria: (1) positive EV71 and/or CA16 genomic RNA; (2) isolation of CA16, EV71 or other EVs; and (3) a fourfold increase in the concentrations of neutralized antibodies against EV71 or other EVs in the late phase rather than in the early phase.

Demographic data of permanent residents in Qingdao were collected from the statistical yearbook and the children's immunization programme each year during the study period. Weekly meteorological information including atmospheric temperature, precipitation, relative humidity, and sunshine hours between 1 January 2007 and 31 December 2014 was obtained from Qingdao Meteorological Bureau.

\section{Sample collection}

A total of 18 sentinel hospitals are responsible for HFMD surveillance in Qingdao. From 1 January 2009 , throat swabs were randomly collected from at least five mild cases every month from April to September and from all mild cases in the other months in each sentinel hospital; while swabs of severe cases were collected in Qingdao Children's Hospital, the designated tertiary hospital responsible for the diagnosis and treatment of all severe HFMD cases in this city. All swab samples were stored at $4{ }^{\circ} \mathrm{C}$ immediately after collection and quickly sent to the national network laboratory in Qingdao for aetiological identification.

\section{Aetiological identification}

In the network laboratory, technicians extracted viral RNA from the throat swabs using MagNA Pure LC total nucleic acid isolation kits in an automated nucleic acid extraction instrument (MagNA Pure LC 2.0; Hoffmann-La Roche, Switzerland) according to the manufacturer's instruction. The extracted nucleic acids were dissolved in $50 \mu \mathrm{l}$ RNase-free distilled water and preserved at $-80^{\circ} \mathrm{C}$. Fluorescence reverse transcription-polymerase chain reaction (RT-PCR) nucleic acid detection kits (Jinhao, China and Zhijiang, China) were applied to detect pan-EVs, EV71, and CA16. For specimens positive for pan-EVs but negative for EV71 and CA16 nucleic acids, semi-nested RT-PCR was used to amplify the VP1 gene of EVs. The PCR amplicons were routinely sequenced. The sequence fragments were connected using Sequencher software (www.genecodes.com/). The genotypes of EVs were determined by online BLAST (blast.ncbi.nlm.nih.gov/Blast.cgi).

\section{Statistical analysis}

To determine the effect of meteorological factors on the HFMD epidemic we conducted descriptive analysis and correlation analysis, respectively. In the descriptive analysis, we matched the weekly HFMD incidence in children aged $0-5$ years with weekly averaged atmospheric temperature, relative humidity, and 
Table 1. The HFMD cases reported via NNDRS in Qingdao, China, 2007-2014

\begin{tabular}{|c|c|c|c|c|c|c|}
\hline \multirow[b]{2}{*}{ Year } & \multirow[b]{2}{*}{$\begin{array}{l}\text { HFMD } \\
\text { cases }\end{array}$} & \multirow[b]{2}{*}{$\begin{array}{l}\text { Sex ratio } \\
\text { (male/female) }\end{array}$} & \multicolumn{2}{|c|}{$\begin{array}{l}\text { Annual incidence rate } \\
\text { (per } 100000)\end{array}$} & \multirow{2}{*}{$\begin{array}{l}\text { Ratio of } \\
\text { cases aged } \\
0-5 \text { years/ } \\
\text { all cases }(\%)\end{array}$} & \multirow[b]{2}{*}{$\begin{array}{l}\text { Number of } \\
\text { severe cases* }\end{array}$} \\
\hline & & & $\begin{array}{l}\text { Whole } \\
\text { population }\end{array}$ & $\begin{array}{l}\text { Population } \\
(0-5 \text { years })\end{array}$ & & \\
\hline 2007 & 8019 & $1 \cdot 57$ & $103 \cdot 75$ & $1179 \cdot 86$ & 88 & - \\
\hline 2008 & 2446 & $1 \cdot 57$ & $31 \cdot 51$ & $364 \cdot 42$ & 88 & - \\
\hline 2009 & 15323 & $1 \cdot 47$ & $196 \cdot 83$ & $2352 \cdot 07$ & 90 & 643 \\
\hline 2010 & 14725 & $1 \cdot 60$ & 188.68 & $2378 \cdot 47$ & 93 & 535 \\
\hline 2011 & 9073 & $1 \cdot 62$ & $104 \cdot 11$ & $1618 \cdot 65$ & 91 & 714 \\
\hline 2012 & 11158 & $1 \cdot 61$ & $127 \cdot 02$ & $2121 \cdot 47$ & 89 & 248 \\
\hline 2013 & 6043 & $1 \cdot 59$ & $64 \cdot 66$ & $1363 \cdot 08$ & 90 & 449 \\
\hline 2014 & 11854 & $1 \cdot 48$ & $132 \cdot 41$ & $2270 \cdot 00$ & 90 & 1073 \\
\hline Total & 78641 & $1 \cdot 55$ & $117 \cdot 58$ & $1691 \cdot 12$ & 90 & 3662 \\
\hline
\end{tabular}

NNDRS, National Notifiable Disease Reporting System.

* In 2007 and 2008, standardized classification criteria were not implemented.

sunshine hours from calendar weeks $1-52$ between 1 January 2007 and 31 December 2014, respectively. In the correlation analysis, the Shapiro-Wilk test was initially used to examine the distribution of weekly meteorological data in the 8 years. If the weekly data were not normally distributed, Spearman rank correlation analysis was applied to examine the correlation between each meteorological variable and HFMD incidence in children aged $0-5$ years. $\chi^{2}$ test was applied to determine the difference of categorical variables. All analyses were two-sided and conducted using SPSS v. 19.0 for Windows (IBM Corp., USA). $P<0.05$ was considered as significant.

\section{RESULTS}

\section{Distribution of HFMD cases}

From 1 January 2007 to 31 December 2014, a total of 78641 HFMD cases were reported to the Municipal CDC of Qingdao via NNDRS. Of those cases, 3662 were diagnosed as severe (in 2007 and 2008, the severe cases were not stratified). Annual average HFMD incidence was $117 \cdot 6 / 100000$ in the entire population. Of the reported cases, $71084(90 \cdot 39 \%)$ occurred in children aged $0-5$ years, with an incidence of $1691 \cdot 2 /$ 100 000. The HFMD incidence is higher in males, with an average male/female ratio of 1.55 , ranging from 1.47 in 2009 to 1.62 in 2011 (Table 1). HFMD incidence in children aged $0-5$ years was higher in urban areas including Licang, Shinan, Shibei, and Laoshan than in suburban areas including Huangdao, Jimo, Jiaozhou, Laixi, and Pingdu (Fig. 1). The epidemic intensity differed but the trend was similar. Generally, HFMD incidence in children aged $0-5$ years began to increase from early spring, peaked between spring and summer, decreased gradually in late summer, and became rare in autumn and winter (Fig. 2). The epidemic strength of this disease varied, for example the incidence was extremely low in 2008. However, the tendency of incidence in each season was consistent over the 8 years.

\section{Aetiological characteristics of HFMD}

Nucleic acids were extracted from throat swabs of the HFMD cases for pathogen identification. Of those tested, the positive rates of EVs were from $24 \%$ to $65 \%$ in the 8 years (Table 2). Before 2010, CA16 and EV71 were the major HFMD pathogens identified in Qingdao. Other types of EVs such as CA2, CA4, CA5, CA6, CA8, CA10, CA12, CA14, CB1, $\mathrm{CB} 2, \mathrm{CB} 3, \mathrm{CB} 4, \mathrm{CB} 5$, and E6 accounted for more than half of the HFMD pathogens from 2011 to 2014. EV71 was prone to causing more severe cases than was CA16 $\left(\chi^{2}=46 \cdot 3, \quad P<0.001\right)$ (Table 3 ). However, EV71 infection was not statistically related to severe cases compared to other EV types $\left(\chi^{2}=\right.$ $1 \cdot 013, P=0 \cdot 314)$, indicating that other EV types might also contribute to the severity of HFMD. With the use of relatively intact aetiological data in 2013 and 2014, we found that CA10 caused more severe HFMD cases than did CA6 $\left(\chi^{2}=20 \cdot 49, P<\right.$ $0 \cdot 001)$; CA6 was not significantly related to the severity of HFMD compared to non-CA6 EVs $\left(\chi^{2}=2 \cdot 15\right.$, $P=0 \cdot 143$ ); however, CA10 was significantly more related to the severity compared to non-CA10 EVs 

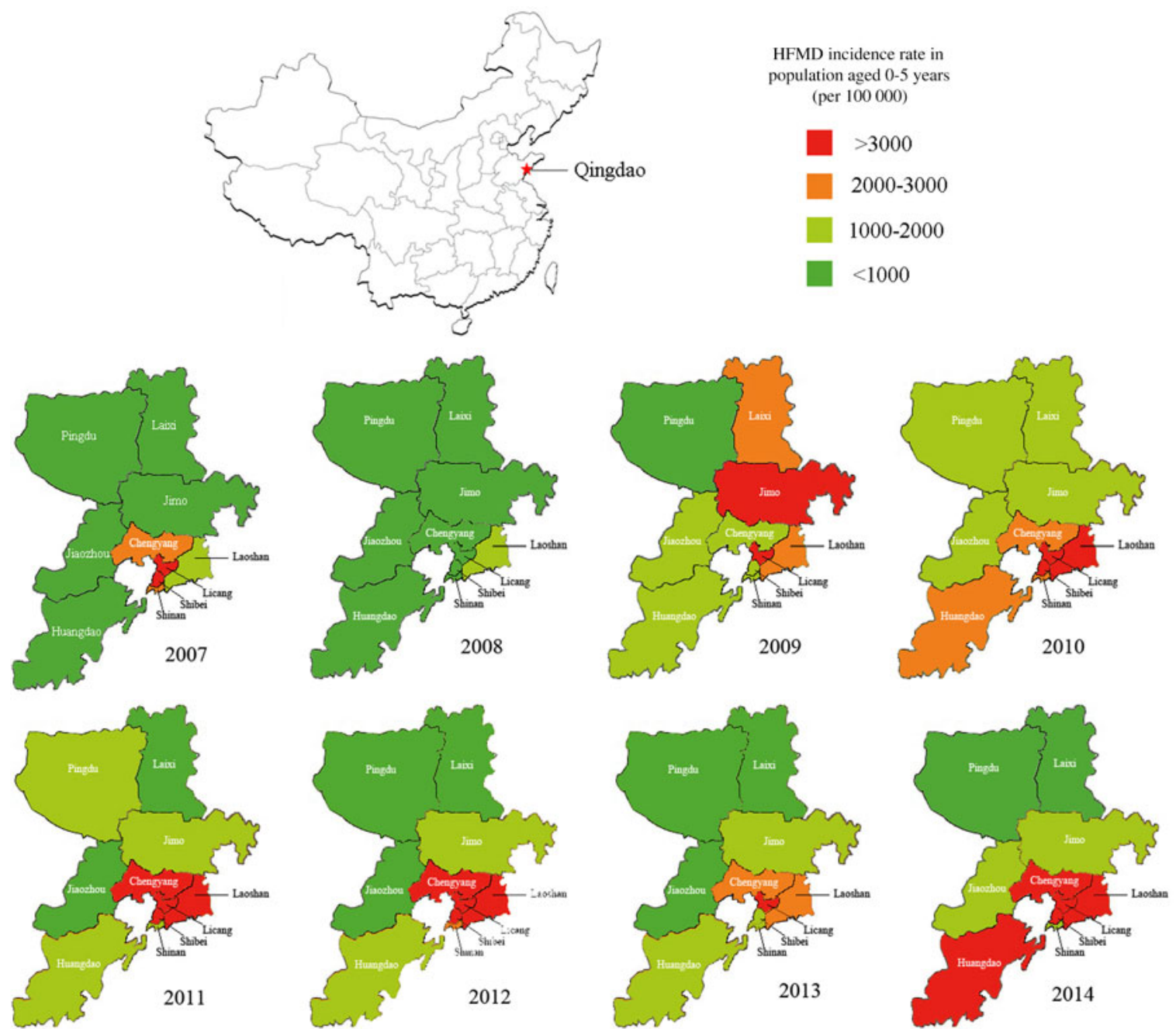

Fig. 1. The distribution of HFMD cases in the population aged $\leqslant 5$ years in each district of Qingdao, China from 2007 to 2014. The epidemic covered all ten districts in Qingdao and mainly attacked urban districts (Shinan, Shibei, Licang, Chengyang, Laoshan).

$\left(\chi^{2}=41 \cdot 01, \quad P<0 \cdot 001\right)$. By summarizing the aetiological data from 2009 to 2014, we found that EV71 was more frequently identified in the off-peak months (September-May) than in the peak months (JuneAugust), either for all HFMD cases or for severe cases (Fig. 3).

\section{Onset locations of HFMD cases}

We summarized the data of locations where HFMD occurred from 2009 to 2014 . We found that $99 \cdot 42 \%$ of the HFMD cases occurred in communities, nurseries, and elementary schools. Community-derived patients accounted for $65 \cdot 11 \%$ of the entire HFMD cases, followed by nursery-derived cases (34.11\%). The ratios of HFMD occurrence in nurseries were consistent from 2009 to 2012 but greatly decreased in 2013 and 2014 (Table 4). The cases found in communities accounted for $>50 \%$ of all patients each year, with the highest percentages in 2013 and 2014.

\section{Association of meteorological variables with HFMD occurrence}

As HFMD occurred periodically each year (Fig. 2), it was necessary to evaluate if meteorological variables were the major determinants of the HFMD epidemic. We matched the weekly HFMD incidence in children aged $0-5$ years with the weekly meteorological variables from calendar weeks 1-52 between 2007 and 2014. We found that the HFMD incidence increased with increasing atmospheric temperature but reached 


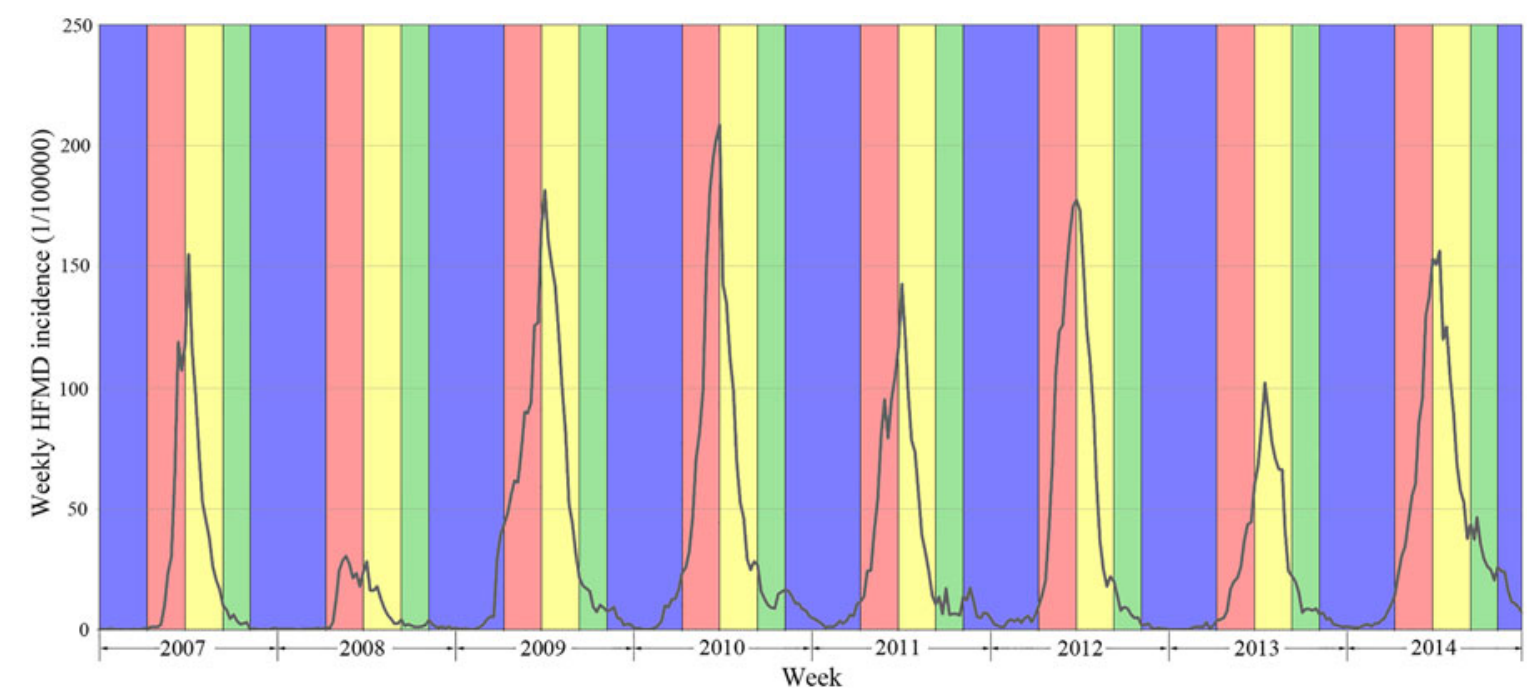

Fig. 2. Weekly HFMD incidence in the population aged $\leqslant 5$ years in Qingdao from 1 January 2007 to 31 December 2014. Light red indicates spring; yellow, summer; green, autumn; blue, winter.

Table 2. Aetiological composition of all HFMD cases in Qingdao, China, 2007-2014

\begin{tabular}{|c|c|c|c|c|c|}
\hline \multirow[b]{2}{*}{ Year } & \multirow[b]{2}{*}{$\begin{array}{l}\text { Cases } \\
\text { examined }\end{array}$} & \multirow[b]{2}{*}{$\begin{array}{l}\text { Positive } \\
\text { cases } n(\%)\end{array}$} & \multicolumn{3}{|c|}{ Aetiological composition, $n(\%)$} \\
\hline & & & EV71 & CA16 & $\begin{array}{l}\text { Other } \\
\text { enteroviruses* }\end{array}$ \\
\hline 2007 & 291 & $90(31)$ & $15(17)$ & $75(83)$ & - \\
\hline 2008 & 1328 & $405(30)$ & $145(36)$ & $260(64)$ & - \\
\hline 2009 & 2470 & $597(24)$ & $455(76)$ & $45(8)$ & $97(16)$ \\
\hline 2010 & 1613 & $1023(63)$ & $431(42)$ & 418 (41) & $174(17)$ \\
\hline 2011 & 1976 & $750(38)$ & $261(35)$ & $24(3)$ & $465(62)$ \\
\hline 2012 & 976 & $300(31)$ & $49(16)$ & 56 (19) & $195(65)$ \\
\hline 2013 & 980 & $636(65)$ & $272(43)$ & $60(9)$ & $304(48)$ \\
\hline 2014 & 1703 & $910(53)$ & $316(35)$ & 257 (28) & $337(37)$ \\
\hline
\end{tabular}

* In 2007 and 2008, other enteroviruses were not fully examined.

Table 3. Aetiological composition of severe HFMD in Qingdao, China, 2009-2014

Aetiological composition, $n(\%)$

\begin{tabular}{|c|c|c|c|c|c|}
\hline Year & $\begin{array}{l}\text { Severe cases } \\
\text { examined }\end{array}$ & $\begin{array}{l}\text { Positive } \\
\text { cases } n(\%)\end{array}$ & EV71 & CA16 & $\begin{array}{l}\text { Other } \\
\text { enteroviruses }\end{array}$ \\
\hline 2009 & 502 & $124(25)$ & $74(60)$ & $10(8)$ & $40(32)$ \\
\hline 2010 & 485 & $331(68)$ & $206(62)$ & $48(15)$ & $77(23)$ \\
\hline 2011 & 631 & $221(35)$ & $51(23)$ & $4(2)$ & $166(75)$ \\
\hline 2012 & 248 & $78(31)$ & $27(35)$ & $11(14)$ & $40(51)$ \\
\hline 2013 & 464 & $269(58)$ & $105(39)$ & $11(4)$ & $153(57)$ \\
\hline 2014 & 1073 & $512(48)$ & $171(34)$ & $109(21)$ & $232(45)$ \\
\hline
\end{tabular}

the peak 5 weeks before the temperature reached the maximum; the levels of precipitation and relative humidity were also positively related to the HFMD epidemic; whereas sunshine hours seemed to be inversely related to the HFMD epidemic (Fig. 4). We then evaluated the statistical correlation of the weekly HFMD incidence in children aged $0-5$ years with each of the weekly meteorological variables. The Shapiro-Wilk 

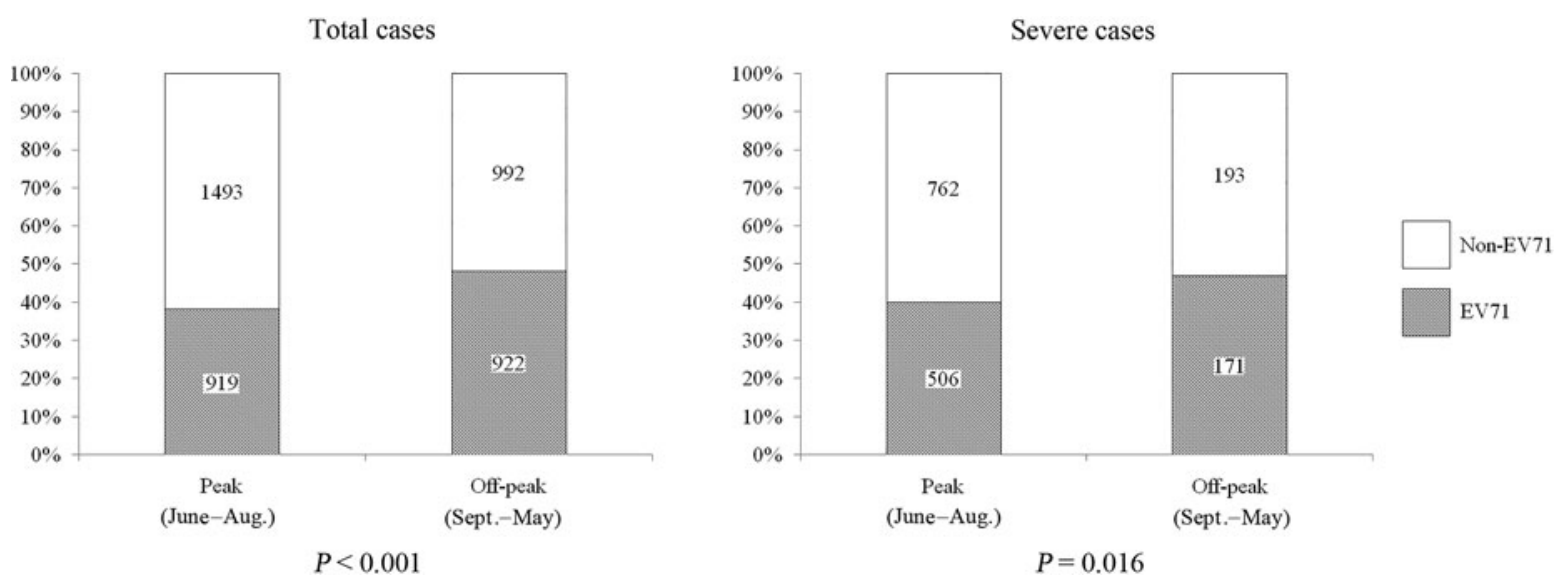

Fig. 3. Proportions of EV71 and non-EV71 enteroviruses identified in all HFMD cases and severe cases during the off-peak months (September-May) and the peak months (June-August) in Qingdao, 2009 to 2014.

Table 4. Onset places of HFMD cases in Qingdao, China, 2009-2014

\begin{tabular}{lrlllr}
\hline \hline Year & Total & Nursery $n(\%)$ & Community $n(\%)$ & School $n(\%)$ & Others $n(\%)$ \\
\hline 2009 & 15323 & $5189(33 \cdot 86)$ & $9382(61 \cdot 23)$ & $694(4 \cdot 53)$ & $58(0 \cdot 38)$ \\
2010 & 14715 & $5561(37 \cdot 80)$ & $8651(58 \cdot 79)$ & $462(3 \cdot 14)$ & $41(0 \cdot 28)$ \\
2011 & 9073 & $3831(42 \cdot 22)$ & $4780(52 \cdot 68)$ & $407(4 \cdot 49)$ & $55(0 \cdot 61)$ \\
2012 & 11158 & $4159(37 \cdot 27)$ & $6302(56 \cdot 48)$ & $597(5 \cdot 35)$ & $100(0 \cdot 90)$ \\
2013 & 6043 & $1457(24 \cdot 11)$ & $4247(70 \cdot 28)$ & $274(4 \cdot 53)$ & $65(1 \cdot 08)$ \\
2014 & 11854 & $3582(30 \cdot 22)$ & $7638(64 \cdot 43)$ & $556(4 \cdot 69)$ & $78(0 \cdot 66)$ \\
\hline \hline
\end{tabular}

test indicated that none of these meteorological variables was normally distributed. Spearman rank correlation analysis showed that HFMD incidence in children aged $0-5$ years was positively correlated with atmospheric temperature $\left(r_{s}=0.77, P<0.001\right)$, relative humidity $\left(r_{s}=0.507, P<0.001\right)$, and precipitation $\left(r_{s}=0.328, P<0.001\right)$. However, the incidence rate was not correlated with sunshine hours $\left(r_{s}=\right.$ $0.014, P=0 \cdot 781)$. Thus, the rise in atmospheric temperature, precipitation, or relative humidity facilitates the HFMD epidemic.

\section{DISCUSSION}

In this study, we selected Qingdao, a tourist port city with four distinctive seasons to evaluate the effects of meteorological factors on the HFMD epidemic. The results indicated that HFMD cases mainly occurred from early spring to early summer in Qingdao. Of the reported cases during the 8 years, $90.39 \%$ occurred in children aged $0-5$ years. We reported that HFMD incidence in children aged $0-5$ years in Ningbo, a warmer port city in East China, was 3066.8/100 000 between 2009 and 2011 [8]. The incidence was 2138.3/100 000 in Qingdao between 2009 and 2011 (Table 1). Thus, HFMD occurs more frequently in children living in warmer cities. HFMD incidence was higher in urban areas than in suburban areas, indicating that living in crowded conditions and/or frequently sharing public facilities may facilitate the transmission of EVs [15]. Interestingly, HFMD incidence was extremely low in 2008 when the aquatic sports of the 2008 Summer Olympics was held in Qingdao. The local government temporarily reinforced prophylactic measures like sterilization of children's environments to control HFMD, at a very high cost. To establish routine prophylaxes cost-effectively, it is necessary to clarify the major risk factors of the HFMD epidemic.

By summarizing the meteorological data within the 8 years, we found that the rise in atmospheric temperature, precipitation, or relative humidity was positively correlated to the HFMD epidemic; whereas sunshine hours might be inversely related to the HFMD epidemic in children aged $0-5$ years. Spearman rank correlation analysis indicated that 

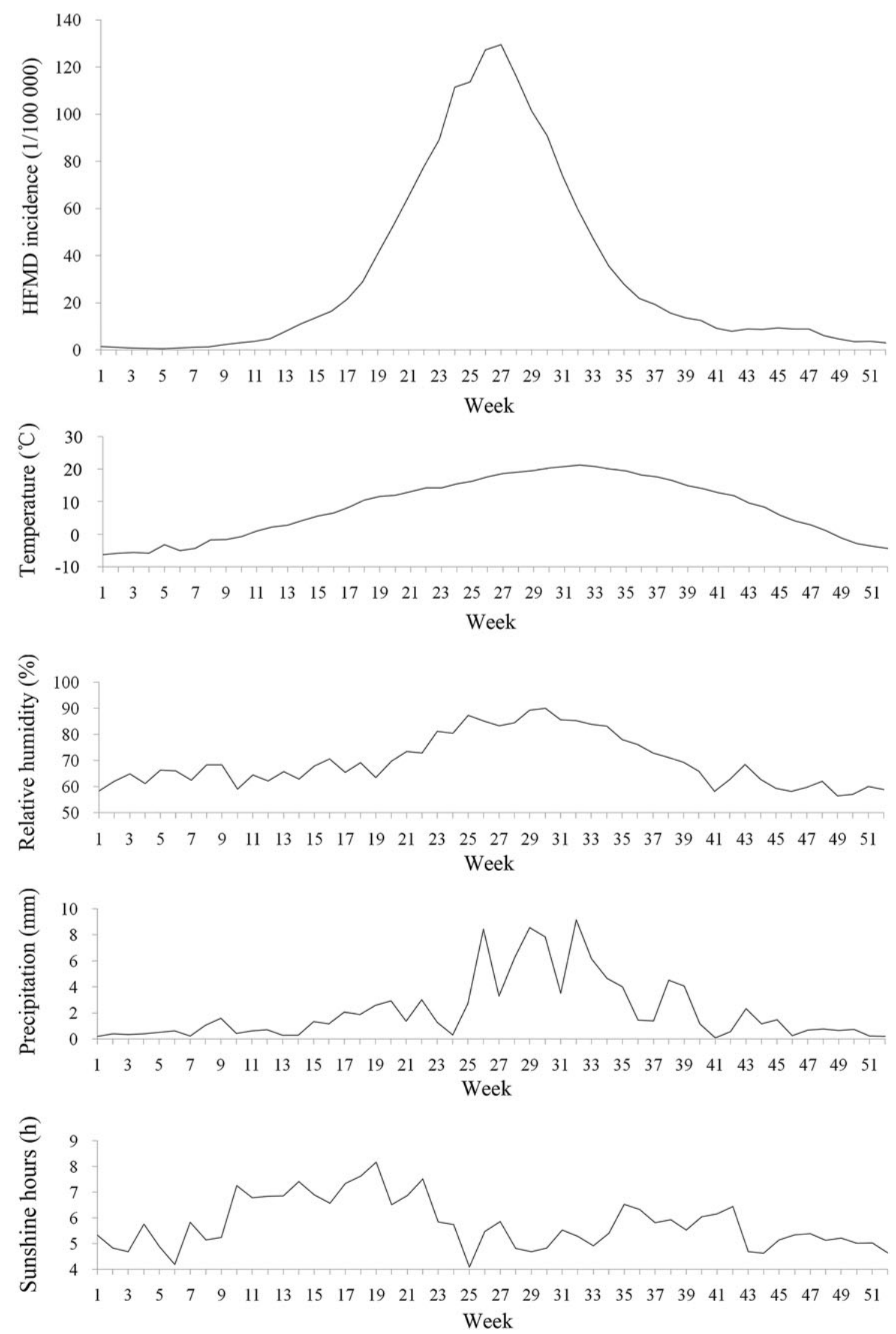

Fig. 4. Correlations of HFMD that occurred in the population aged $\leqslant 5$ years with the major meteorological variables in each week by averaging weekly data from 2007 to 2014 . 
the incidence rate was positively correlated with atmospheric temperature, relative humidity, and precipitation. This can be explained in four aspects. First, children increase outdoor activity when it becomes warmer from early spring to early summer, thus facilitating transmission of the disease. HFMD incidence is higher in male children, with an average male/ female ratio of $1 \cdot 55$, possibly because male children are more active. Second, EVs are highly contagious in warm and humid weather, while precipitation can increase relative humidity. Third, frequent precipitation and subsequent flooding may damage the barriers between clean and dirty living environments, especially in suburban areas where some public toilet facilities are not well administered, thus causing water pollution. Fourth, although sunlight can inactivate human EVs efficiently [16], the HFMD-causing EVs are highly contagious in conditions not exposed to sunlight. In addition, we found that HFMD incidence in children aged $0-5$ years increased with increasing atmospheric temperature but reached its peak at week 27 even if the temperature continued to increase (Fig. 4). This mismatch should be caused by public health interventions. Local governments usually take steps to block the transmission pathways if incidence increases rapidly.

The aetiological investigation indicated that EV71 and CA16 were the major aetiological agents of HFMD in 2009 and 2010; however, other EVs accounted for $>50 \%$ of those in 2011 and 2012. This might be partially attributable to immune protection. Although EV71 vaccine has not been administered, the immunization barrier against EV71 should be gradually established because EV71 has been epidemic for a number of years in Qingdao. We have previously reported that the prevalence of neutralization antibody against EV71 increased with the increasing age of residents [8]. It has been proven that inactivated EV71 vaccine has good safety and immunogenicity in infants and confers a relatively high rate of protection against EV71-related diseases [17]. As immune pressure for EV71 increases in affected populations, other EVs may take its place and become the dominant pathogens of HFMD. This may explain the co-circulation of aetiological agents of HFMD in consecutive years. CA6 has been linked to severe outbreaks worldwide since 2008 and associated with severe HFMD in children and adults [18, 19]. CA6 has become a major strain for HFMD in southern China $[20,21]$. CA10 was not a major strain for HFMD in China but its proportion as the pathogen has increased recently [20-22]. Interestingly, by summarizing the aetiological data in this study, we found that CA10, rather than CA6, was prone to causing severe HFMD. Although the severity of HFMD is related to multiple factors [8], the pathogen should be the most important one. Our findings might reduce the urgency and uniqueness of EV71 vaccination in the fight against severe HFMD. Public health prophylaxes focusing on CA10 and EV71 should become a priority.

To establish a disease outbreak, three prerequisites are needed: (i) the presence of a pathogen in sufficient quantities, (ii) an appropriate mode of transmitting the pathogen, and (iii) an adequate pool of susceptible persons exposed to the pathogen. In this study, we found that HFMD outbreaks started in early May and peaked between spring and summer, indicating that the three prerequisites are all met in the peak months. In addition, we found that HFMD cases occurred mostly in communities. It is highly likely that large amounts of the pathogen are normally carried by adults caring for infants, especially by older age groups. Because of the high positive rate of circulating neutralizing antibody against EV71 in adults [8], the adults with neutralizing antibody might be asymptomatic carriers for EVs. EVs can infect susceptible infants when the infants become active in warm and unsanitary conditions. Future directions of controlling the HFMD epidemic should focus on communities with backward public health infrastructures. Interestingly, EV71 was less frequent in the peak months than in the off-peak months (Fig. 3). This can be explained by the fact that EV71 frequently causes severe notifiable medical condition in the offpeak months.

The outcomes of this study should be integrated into the current programme for the prophylaxis and control of the HFMD epidemic. Hygiene education should be implemented in community-based populations especially for families with children and/or children's guardians before atmospheric temperature, relative humidity, and precipitation rise. Public sanitary control including disinfection measures can be precisely conducted in light of climate changes. EVs such as CA10 and EV71 that can cause severe HFMD cases should be monitored in communities with backward public health infrastructures.

In summary, HFMD is a major public health issue for children aged $0-5$ years, with an incidence of $1691 \cdot 2 / 100000$ in Qingdao, a large tourist port city in northern China. Atmospheric temperature, precipitation, and relative humidity positively correlate with 
the HFMD epidemic. EV71 is prone to causing more severe cases than is CA16. CA10 also causes more severe HFMD cases than does CA6. CA10, compared to non-CA10 EVs, is significantly more related to the severity of this disease. To prevent and control the HFMD epidemic cost-effectively, climate changes can be integrated into the current prophylactic programme for HFMD. Co-circulation of severe HFMD-causing agents such as CA10 and EV71 should be regularly monitored in communities with backward public health infrastructures.

\section{ACKNOWLEDGMENTS}

The authors acknowledge Mr Yi-bo Ding, Dr Zi-xiong Li, Dr Xiao-mei Hou, Ms. Chong Ni, Dr Jian-hua Yin, Ms. Lucrece Hazoume, Dr Xiao-jie Tan, Professor Hong-wei Zhang, and colleagues in district CDCs and network laboratories for their collaboration in laboratory work, field survey, and sample collection. This work was supported by the General Logistics of PLA in China (G.W.C., grant no. AWS11L009) and Science and Technology Bureau of Qingdao (F.C.J., grant no. 10-3-3-8-2-nsh).

\section{DECLARATION OF INTEREST}

None.

\section{REFERENCES}

1. Blomqvist $\mathbf{S}$, et al. Co-circulation of coxsackieviruses A6 and A10 in hand, foot and mouth disease outbreak in Finland. Journal of Clinical Virology 2010; 48: 49-54.

2. Itagaki A, et al. A clustering outbreak of hand, foot, and mouth disease caused by Coxsackie virus A10. Microbiology and Immunology 1983; 27: 929-935.

3. Puenpa J, et al. Molecular characterization and complete genome analysis of human enterovirus 71 and coxsackievirus A16 from children with hand, foot and mouth disease in Thailand during 2008-2011. Archives of Virology 2011; 156: 2007-2013.

4. Liu CC, et al. An outbreak of enterovirus 71 infection in Taiwan, 1998: epidemiologic and clinical manifestations. Journal of Clinical Virology 2000; 17: 23-30.

5. Zhang Y, et al. An emerging recombinant human enterovirus 71 responsible for the 2008 outbreak of hand foot and mouth disease in Fuyang city of China. Journal of Virology 2010; 7: 94.

6. Zhang Y, et al. An outbreak of hand, foot, and mouth disease associated with subgenotype $\mathrm{C} 4$ of human enterovirus 71 in Shandong, China. Journal of Clinical Virology 2009; 44: 262-267.

7. Mao LX, et al. Epidemiology of hand, foot, and mouth disease and genotype characterization of enterovirus 71 in Jiangsu, China. Journal of Clinical Virology 2010; 49: 100-104.

8. Ni H, et al. Epidemiological and etiological characteristics of hand, foot, and mouth disease in Ningbo, China, 2008-2011. Journal of Clinical Virology 2012; 54: 342 348.

9. Wei J, et al. The effect of meteorological variables on the transmission of hand, foot and mouth disease in four major cities of shanxi province, China: a time series data analysis (2009-2013). PLoS Neglected Tropical Diseases 2015; 9: e0003572.

10. Zhang W, et al. Quantifying the adverse effect of excessive heat on children: an elevated risk of hand, foot and mouth disease in hot days. Science of the Total Environment 2015; 541: 194-199.

11. Liu W, et al. Spatiotemporal dynamics of handfoot-mouth disease and its relationship with meteorological factors in Jiangsu Province, China. PLoS ONE 2015; 10: e0131311.

12. Liu Y, et al. Spatio-temporal analysis of the relationship between climate and hand, foot, and mouth disease in Shandong province, China, 2008-2012. BMC Infectious Diseases 2015; 15: 146.

13. Liu L, et al. Spatio-temporal clustering of hand, foot and mouth disease at the county level in Sichuan province, China, 2008-2013. Epidemiology \& Infection 2015; 143: 831-838.

14. Li T, et al. Hand-foot-and-mouth disease and weather factors in Guangzhou, southern China. Epidemiology \& Infection 2014; 142: 1741-1750.

15. Xie YH, et al. Important roles of public playgrounds in the transmission of hand, foot, and mouth disease. Epidemiology \& Infection 2015; 143: 1432-1441.

16. Fujioka RS, Yoneyama BS. Sunlight inactivation of human enteric viruses and fecal bacteria. Water Science and Technology 2002; 46: 291-295.

17. Liang ZL, et al. Progress on the research and development of inactivated EV71 whole-virus vaccines. Human Vaccines and Immunotherapeutics 2013; 9: 1701-1705.

18. Downing C, et al. Coxsackievirus A6 associated hand, foot and mouth disease in adults: clinical presentation and review of the literature. Journal of Clinical Virology 2014; 60: 381-386.

19. Bruning AH, et al. An atypical course of coxsackievirus A6 associated hand, foot and mouth disease in extremely low birth weight preterm twins. Journal of Clinical Virology 2015; 65: 20-22.

20. Guo WP, et al. Fourteen types of co-circulating recombinant enterovirus were associated with hand, foot, and mouth disease in children from Wenzhou, China. Journal of Clinical Virology 2015; 70: 29-38.

21. Xu M, et al. Genotypes of the enterovirus causing hand foot and mouth disease in Shanghai, China, 2012-2013. PLoS ONE. Published online: 23 September 2015. doi:10.1371/journal.pone.0138514.

22. Zhang C, et al. Phylogenetic analysis of the major causative agents of hand, foot and mouth disease in Suzhou city, Jiangsu province, China, in 2012-2013. Emerging Microbes and Infections 2015; 4: e12. 